\title{
A Novel Mutation in the CLDN16 Gene in a Palestinian Family with Familial Hypomagnesemia with Hypercalciuria and Nephrocalcinosis
}

\author{
Abdulsalam Abu-Libdeh ${ }^{1} \quad$ Bassam Abu-Libdeh $^{1} \quad$ Ulla Najwa Abdulhag ${ }^{1}$ \\ ${ }^{1}$ Department of Pediatrics, Makassed Islamic Hospital, \\ Jerusalem, Israel \\ Address for correspondence Abdulsalam Abu-Libdeh, MD, \\ Department of Pediatrics, Makassed Islamic Hospital, P.O.Box 19482, \\ Mount of Olives, Jerusalem, Israel \\ J Child Sci 2017;7:e32-e35. \\ (e-mail: draabulibdeh@hotmail.com).
}

\begin{abstract}
Familial hypomagnesemia with hypercalciuria and nephrocalcinosis (FHHNC) is a rare autosomal recessive renal disorder characterized by excessive renal magnesium and calcium loss, bilateral nephrocalcinosis, and progressive renal failure, due to impaired tubular reabsorption in the thick ascending loop of Henle. FHHNC is caused by loss of function mutations in the claudin-16 gene (CLDN16) and claudin-19 gene (CLDN19). A 2-month-old male infant presented with convulsions during hypomagnesemia,

Keywords

- CLDN16

- CLDN19

- familial hypomagnesemia with hypercalciuria and nephrocalcinosis hypocalcemia, and hypophosphatemia, biochemical findings were consistent with FHHNC. There is a positive family history of the death of a 12 years old sibling due to renal failure. Gene sequencing of the CLDN16 revealed a novel missense mutation with the replacement of $\mathrm{T}$ by $\mathrm{C}$ in codon 120 located in exon 2, predicting cysteine to arginine substitution p.Cys120Arg. This is the first description of this missense mutation and the first confirmation of FHHNC by molecular testing in a Palestinian family which enables genetic counseling and future prenatal diagnosis.
\end{abstract}

\section{Introduction}

Familial hypomagnesemia with hypercalciuria and nephrocalcinosis (FHHNC) is a rare progressive autosomal recessive disease, characterized by hypomagnesemia, hypercalciuria, and nephrocalcinosis. Recently, mutations in the genes encoding tight junctions (TJ) proteins claudin-16 (CLDN16) and claudin-19 (CLDN19) were found to be responsible for this renal tubular disorder. ${ }^{1}$ The main clinical features of FHHNC include hypomagnesemia, hypercalciuria, polydipsia polyuria, peritubular calcifications, and/or renal stones in early childhood.

Mutations in the CLDN16 and CLDN19 genes have been linked to FHHNC. The expression of CLDN16 and CLDN19 occurs at the ascending limb of the nephron. Both claudins interact and form a cation-selective TJ paracellular channel. ${ }^{2}$ Mutant forms of both CLDN16 and CLDN19 lead to disruption of this interaction which underlies the pathomechanism of FHHNC. ${ }^{1}$ To date, 54 different CLDN16 mutations have been identified, ${ }^{3}$ most of these mutations (67\%) were missense mutations, although deletions and frameshift mutations were also reported. ${ }^{4}$

Here, we describe a novel missense mutation in the CLDN16 gene in a Palestinian family with FHHNC.

\section{Case Report}

A 2-month-old male infant, born to healthy consanguineous Palestinian parents, presented with a history of hypomagnesemia convulsions that started at the age of 40 days ( - Fig. 1 ). His investigations revealed hypomagnesemia and hypocalcemia along with a positive urine culture, and evidence of nephrocalcinosis on renal ultrasound but without hydronephrosis. Parathyroid hormone was elevated $(240 \mathrm{pg} / \mathrm{mL})$ and fractional excretion of magnesium was high (52\%). Also, the 24hour urine collection for calcium was high $(14 \mathrm{mg} / \mathrm{kg} / \mathrm{d})$. Kidney function tests were within the normal range for his age. Voiding cystourethrogram revealed no abnormalities. Ophthalmic examination and brain computed tomography received

March 13, 2017

accepted after revision

June 19, 2017
DOI https://doi.org/

$10.1055 / \mathrm{s}-0037-1604294$. ISSN 2474-5871.
Copyright @ 2017 Georg Thieme Verlag

KG Stuttgart · New York

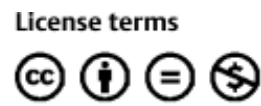




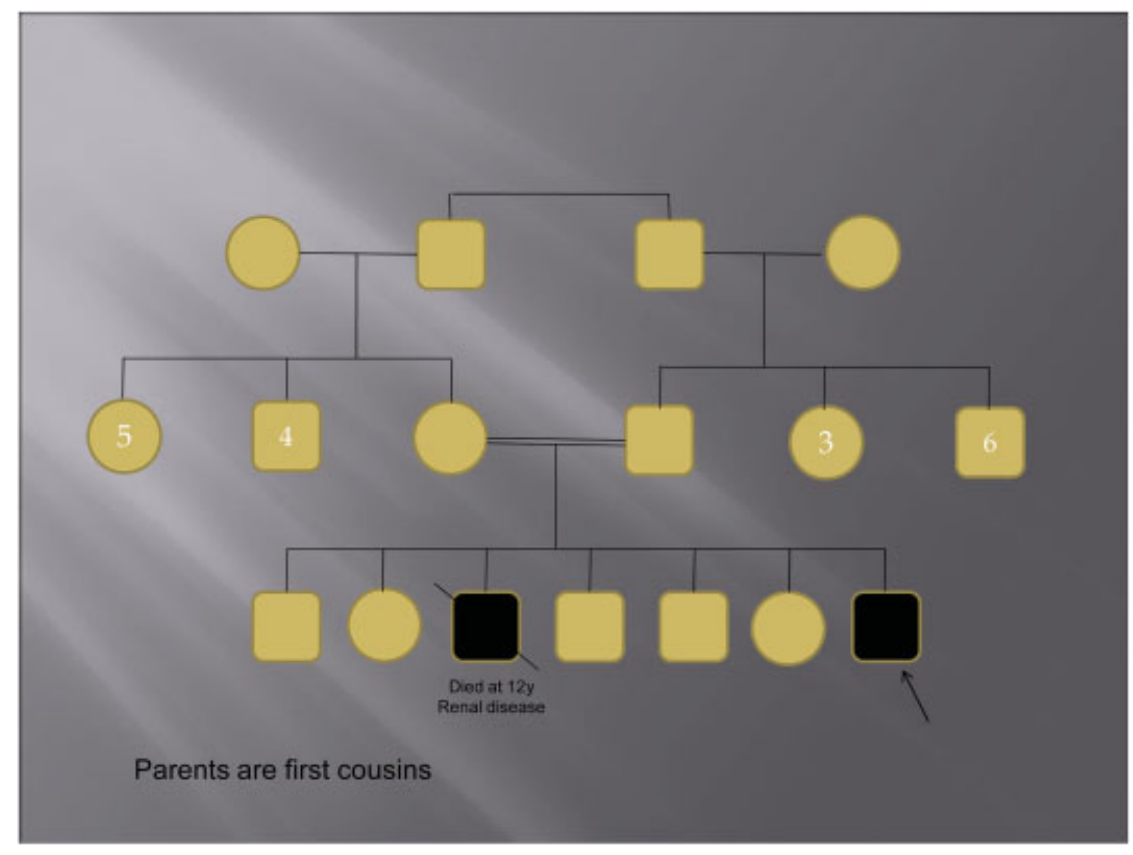

Fig. 1 Family pedigree showing consanguinity, affected patient, and affected brother.

scan were normal. His initial laboratory results were as shown in - Table 1. The family history was notable for an older sibling who died at the age of 12 years secondary to renal disease.

The clinical picture, together with the laboratory findings, imaging studies, and genetic testing were consistent with FHHNC. He was managed with thiazides and magnesium sulfate intravenously which was then shifted to magnesium citrate orally, with significant improvement of the hypomagnesemia followed by the correction of his hypocalcemia and hyperphosphatemia (see - Table 1).

\section{Sequencing of the CLDN16 Gene}

Before genetic studies, informed consent was obtained from the study participants or their guardians. The study was approved by the Institutional Review Boards of Makassed Islamic Hospital. Genomic DNA was extracted from the whole blood of the patient and family members, using the MagNA Compact System Kit (Roche Diagnostics GmbH, Germany). Genomic DNA was amplified using a PTC 225 DNA Engine (MJ Research Hercules, United States). For the polymerase chain reaction (PCR) reaction $50 \mathrm{ng}$ of DNA from either the proband or other family members was added to a reaction buffer that included $10 \mathrm{pmol}$ of each primer corresponding to the first three exons, $\mathrm{MgCl}_{2}, 1.5 \mathrm{mM}$, dNTPs $0.2 \mathrm{mM}$, PCR $\times 1$ Gold buffer $(15 \mathrm{mM}$ Tris-HCL, pH 8.0, $50 \mathrm{mM} \mathrm{KCL}$ ), and 0.4 unit of AmpliTaq Gold DNA polymerase (both from Applied Biosystems, Foster City, California, United States) in a total volume of $25 \mu \mathrm{L}$. Reaction conditions included an initial 12 -minute denaturation at $95^{\circ} \mathrm{C}$ followed by 37 cycles of 1 minute at $92^{\circ} \mathrm{C}, 1$ minute at $55^{\circ} \mathrm{C}$, and 1 minute at $72^{\circ} \mathrm{C}$ with a final 10 -minute incubation at $72^{\circ} \mathrm{C}$.

Table 1 Table summarizing biochemical findings of our patient in serum and urine

\begin{tabular}{|l|l|l|l|}
\hline Test & Result before treatment & Result after treatment & Normal range \\
\hline $\mathrm{Ca}$ & 5.9 & 9.1 & $9.2-11 \mathrm{mg} / \mathrm{dL}(\mathrm{low})$ \\
\hline $\mathrm{PO}_{4}$ & 8.2 & 6.4 & $4.5-6.7 \mathrm{mg} / \mathrm{dL}(\mathrm{high})$ \\
\hline $\mathrm{Mg}$ & 0.8 & 1 & $1.6-2.6 \mathrm{mg} / \mathrm{dL}(\mathrm{low})$ \\
\hline Parathyroid hormone & 204 & $8.7-79.6 \mathrm{pg} / \mathrm{mL}(\mathrm{high})$ \\
\hline Urea & 8.9 & $6-20 \mathrm{mg} / \mathrm{dL}(\mathrm{normal})$ \\
\hline Creatinine & 0.54 & $0.1-1 \mathrm{mg} / \mathrm{dL}(\mathrm{normal})$ \\
\hline 24-h urine collection & \multicolumn{2}{|l|}{} \\
\hline Glomerular filtration rate & 50 & $35-114 \mathrm{mg} / \mathrm{min} / 1.73 \mathrm{~m}{ }^{2}(\mathrm{normal})$ \\
\hline Calcium & 8.3 & $<4 \mathrm{mg} / \mathrm{kg} / \mathrm{d}(\mathrm{high})$ \\
\hline Others & \multicolumn{2}{l}{} \\
\hline Fractional excretion of Mg & $52 \%$ & High \\
\hline Spot urine calcium/creatinine ratio & 0.9 & High \\
\hline
\end{tabular}


Automated DNA sequencing was performed using the BigDye terminator cycle sequencing chemistry from Applied Biosystems, the ABI PRISM 3700 DNA analyzer, and ABI's data collection software. Results were analyzed with the sequence analysis software and compared with the sequence available from the National Center for Biotechnology Information database (340298).

Primers for amplification and sequencing of the CLDN16 gene were:

\section{Exon 1F: GAAACACACTCAGCCCTTGC R: TGTTGTACATGG CAGTTGGG}

Exon 2F: CAAGGGGAACTGAACTGTGC R: GCACAAAATGG AAACCTGAAC

Exon 3F: TGTCTCTGTGAGTTAATATGGTTCC R: TCACCTCAA GCCCTTAGCAG

Exon 4F: ACCTCTCTGAATCACGCCAG R: TTTGTTTCCACTG GATTGGG

Exon 5F: AAAATAACAGGAAATCTACTTGGC R: GGGAAA GTGTGTGCATAAGG

\section{Results}

Sequencing the coding region of the CLDN16 gene of the affected patient showed a novel missense mutation with the replacement of $\mathrm{T}$ by $\mathrm{C}$ in codon 120 of exon 2 (TGC $\rightarrow$ CGC) predicting cysteine to arginine substitution (p.Cys120Arg) c.358(T > C)/c.T358C ( - Fig. 2A). The parents were heterozygous for this mutation as expected ( - Fig. 2B). No other mutations were found in sequencing the CLDN16 gene (-Fig. 2C).

\section{Discussion}

FHHNC is a rare renal disorder caused by loss of function mutations in the CLDN16 and CLDN19 genes encoding for the renal tight junction proteins CLDN16 and CLDN19.

The clinical phenotype of mutations in CLDN16 is likely to be determined by the location of the mutation within the gene. Mutations affecting amino acids at the extracellular and transmembrane segment cause classical FHHNC, while mutations affecting specific amino acids in the cytosolic domain cause a spontaneously resolving hypercalciuria.

Symptoms of FHHNC usually become evident in the first months of life. Initial symptoms of FHHNC include recurrent urinary tract infections, polyuria, and polydipsia, ${ }^{5}$ in addition to marked hypomagnesemia. In all patients, hypercalciuria and later on nephrocalcinosis are exhibited. Additional findings include nephrolithiasis, abdominal pain, vomiting, convulsions, muscular twitches, failure to thrive, incomplete distal renal tubular acidosis, and hypocitraturia. ${ }^{4}$

The conventional therapy for patients with FHHNC includes magnesium supplements and thiazides to reduce the hypercalciuria, although these medications may improve some biochemical abnormalities, such improvements do not always result in delayed progression of renal dysfunction. ${ }^{5}$ Zimmermann et al studied the efficacy of hydrochlorothiazide (HCT) in FHHNC patients in a clinical trial, and concluded that HCT is effective in

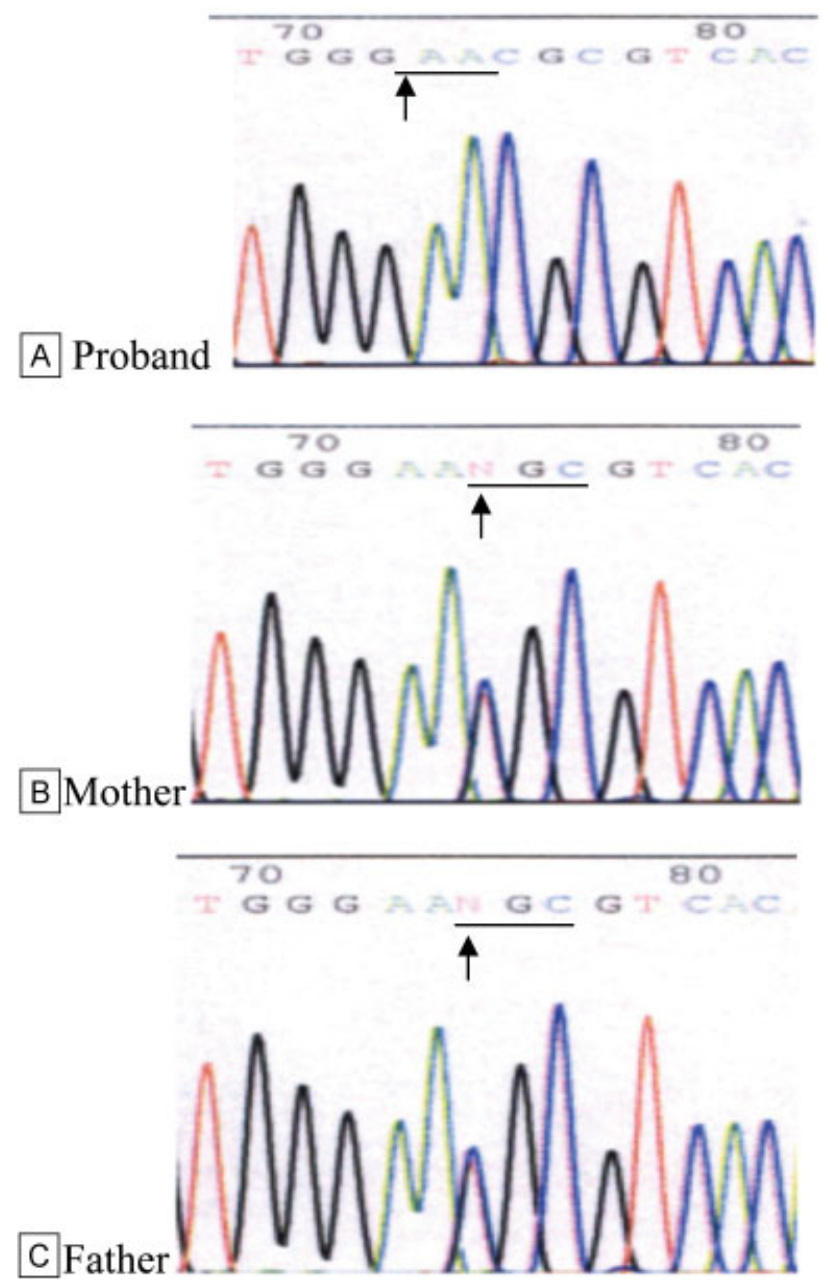

Fig. 2 Linkage analysis of the CLDN16 gene. (A) Mutational analysis of the proband. A missense mutation with the replacement of $T$ by $C$ in codon 120 of exon 2 (arrow), results in the substitution of cysteine to arginine (p.Cys120Arg); (B and C) showing the parents are heterozygous.

reducing hypercalciuria due to CLDN16 mutation on a shortterm basis; however, the efficacy of HCT to attenuate disease progression remains to be evaluated. ${ }^{6}$

No functional studies were performed and we considered the amino acid change in the gene as pathogenic (i.e., mutation) based on the following: (1) positive segregation in the family as parents were heterozygous, (2) the amino acid change is in a region with a predilection to mutation, (3) the changed amino acid is in a conserved residue.

In patients with CLDN16 mutations, hypomagnesemia has been reported to be unresponsive to magnesium administration. ${ }^{5}$ Nevertheless, in our patient, the administration of HCT and magnesium improved his hypomagnesemia significantly followed by a correction of his hypocalcemia and hyperphosphatemia. Here, we have to mention that we report a shortterm follow-up, however, the long-term efficacy of HCT and magnesium administration on the disease progression toward end stage renal disease is to be evaluated in the future.

We conclude that despite the rarity and the intriguing nature of FHHNC disease, it still deserves consideration in any patient presenting with nephrocalcinosis and hypercalciuria, especially in the pediatric age group. In our case, not 
only is this the first time that this novel mutation is described in a Palestinian family, but it also carries a great importance in two aspects. The first one lies in predicting the development of renal failure which was the cause of death in the older sibling. The second aspect is the ability to do genetic counseling for future offsprings with subsequent management.

\section{References}

1 Hou J, Renigunta A, Gomes AS, et al. Claudin-16 and claudin-19 interaction is required for their assembly into tight junctions and for renal reabsorption of magnesium. Proc Natl Acad Sci U S A 2009;106(36):15350-15355
2 Hou J, Renigunta A, Konrad M, et al. Claudin-16 and claudin-19 interact and form a cation-selective tight junction complex. J Clin Invest 2008;118(02):619-628

3 Human Gene Mutation Database (HGMP). Available at http:// www.hgmd.cf.ac.uk/ac/index.php

4 Al-Haggar M, Bakr A, Tajima T, et al. Familial hypomagnesemia with hypercalciuria and nephrocalcinosis: unusual clinical associations and novel claudin 16 mutation in an Egyptian family. Clin Exp Nephrol 2009;13(04):288-294

5 Weber S, Schneider L, Peters M, et al. Novel paracellin-1 mutations in 25 families with familial hypomagnesemia with hypercalciuria and nephrocalcinosis. J Am Soc Nephrol 2001;12(09):1872-1881

6 Zimmermann B, Plank C, Konrad M, et al. Hydrochlorothiazide in CLDN16 mutation. Nephrol Dial Transplant 2006;21(08):2127-2132 